\title{
Dinâmica de sistemas auxiliando na identificação de fatores que podem influenciar em atrasos de entregas e projetos em um instituto de inovação
}

\section{System dynamics assisting in the identification of factors that may influence delivery and project delays at an innovation institute}

DOI: $10.54021 /$ seesv3n1-002

Recebimento dos originais: 05/12/2021

Aceitação para publicação: 05/01/2022

\section{Everton Michels}

Universidade Federal de Santa Catarina

R. Eng. Agronômico Andrei Cristian Ferreira, s/n - Trindade, Florianópolis

E-mail: evertonpmp@gmail.com

\section{Fernando Antonio Fornellini}

Universidade Federal de Santa Catarina

R. Eng. Agronômico Andrei Cristian Ferreira, s/n - Trindade, Florianópolis

E-mail: forcellini@deps.ufsc.br

\section{RESUMO}

Diversos estudos já abordaram o ciclo de retrabalho em dinâmica de sistemas no que tange o gerenciamento de projetos. No entanto, mesmo este servindo para a grande maioria dos casos, algumas especificidades derivam deste modelo, principalmente pelo contexto avaliado. Com base nisso, e diante da dificuldade de gerenciar projetos de inovação, com altos riscos devido ao grau de incertezas, e sofrendo com atraso em entregas e no projeto, este estudo visa identificar quais a variáveis que mais ou menos influenciam nos projetos de inovação do Instituto Senai de Inovação de Sistemas Embarcados. Para tal, foi feito uma revisão tradicional somada a um estudo bibliométrico a fim de embasar este trabalho, junto a um estudo empírico utilizando dinâmica de sistemas, que resultou na identificação das principais variáveis que afetam o sistema de gestão dos projetos do instituto em questão, além de verificar que o mesmo amplia o conhecimento sobre dinâmica de sistemas em projetos e introduz novas variáveis as quais ainda há pouca abordagem.

Palavras-chave: Dinâmica de sistemas, Gerenciamento de projetos, Ciclo de retrabalho, Atraso em projetos, Inovação.

\section{ABSTRACT}

Several studies have addressed the rework cycle in system dynamics with regard to project management. However, even though it can be used in most cases, some specificities derive from this model, mainly due to the evaluated context. Based on this, and facing the difficulty of managing innovation projects, with high risks due to the degree of uncertainties, and suffering with delays in deliveries and in the 
project, this study aims to identify which variables have more or less influence in the innovation projects of the Senai Institute for Innovation in Embedded Systems. To this end, a traditional review was done together with a bibliometric study in order to base this work, together with an empirical study using system dynamics, which resulted in the identification of the main variables that affect the project management system of the institute in question, besides verifying that it expands the knowledge about system dynamics in projects and introduces new variables which there is still little approach.

Keywords: System dynamics, Project management, Rework cycle, Project delay, Innovation.

\section{INTRODUÇÃO}

A aplicação de dinâmica de sistemas no mundo de gerenciamento de projetos já está bem desenvolvida, além de estar em constante melhoria. Prova disso são os diversos trabalhos que surgiram a partir do ciclo de retrabalho em projetos desenvolvido por Cooper (1980). Esta área absorveu de forma positiva os benefícios que a dinâmica de sistemas oferece, e com isso os estudos estão proporcionando cada vez mais conhecimento de valor a dinâmica que está envolvida no gerenciamento de projetos.

No entanto, estes estudos, como é natural, não conseguem atender a todos os casos possíveis, principalmente no que tange a especificidades de cada contexto que pode ser analisado. Desta forma, a necessidade de estudos mais específicos no que se refere a dinâmica em projetos de pesquisa e desenvolvimento (P\&D) com forte viés da inovação é uma questão ainda a ser melhor explorada.

Estudos que buscam explicitar a dinâmica de sistemas na área do gerenciamento de projetos como por exemplo (Cooper, 1980; Ackerman et al., 1997; Ford; Sterman, 1998; Eden et al, 1998, 2000) são trabalhos fundamentais e servem como base para o aprimoramento desta união. Essa união e algumas de suas especificidades como riscos (Williams et al, 1995, 2003; Lyneis et al, 2001; Yi; Xiao, 2008), custos e cronograma (Reichelt; Lyneis, 1999; Yaghootkar; Gil, 2012), P\&D e desenvolvimento de novos produtos (Ottosson; Bjork, 2004; Lin; Chai; Wong; Brombacher, 2008; Akkermans; van Oorschot, 2016, etc.), e outros temas, já mostram-se capazes de explicitar e explicar alguns fenômenos que acontecem nestes sistemas, no entanto, não detalham todos os possíveis, 
principalmente devido a mudanças constantes, bem como as especificidades de cada empresa ou ambiente que ela atua, principalmente quando se trata de projetos de inovação.

Diante disso, este trabalho visa identificar algumas variáveis que podem afetar mais ou menos no atraso de entregas e projetos de pesquisa e desenvolvimento com alto grau de inovação, em um instituto de inovação que trabalha com gerenciamento tanto tradicional quanto ágil, para a gestão de seus projetos.

\section{DINÂMICA DE SISTEMAS NO GERENCIAMENTO DE PROJETOS}

A dinâmica de sistemas na área de gestão de projetos não é algo novo. Roberts (1962) foi o precursor nessa união de áreas, abordando estes temas pela primeira vez juntos. A partir dai outros trabalhos surgiram e continuam sendo tratados, e talvez um dos principais deles tenha sido o estudo sobre ciclo de retrabalho em projetos, desenvolvido por Cooper (1980), e dele outros diversos estudos foram se especializando a fim de melhor entender o ciclo de retrabalho em diversos contextos, como é o caso deste trabalho.

O ciclo de retrabalho, como visto na figura 1, é a característica mais importante de modelos de sistema dinâmicos de projeto. A natureza recursiva do ciclo de retrabalho mostra que o mesmo acaba gerando ainda mais retrabalho, criando assim comportamentos problemáticos que muitas vezes se estendem além da duração do projeto e são a fonte de muitos desafios no gerenciamento de projetos (Cooper, 1980).

A partir do ciclo de retrabalho de Cooper (1980), outros trabalhos foram derivados, e aprimoraram ainda mais os estudos. Este estudo além de utilizar como base o trabalho de Cooper (1980), também buscou na literatura alguns estudos para fornecer uma estrutura coesa que sustentasse algumas das variáveis de aplicação e a dinâmica das mesmas, pois estas servirão de base para este trabalho, além deste estudo fornecer questões que complementam ainda mais os modelos base. 
fig. 1. ciclo de retrabalho (adaptado de cooper, 1983)

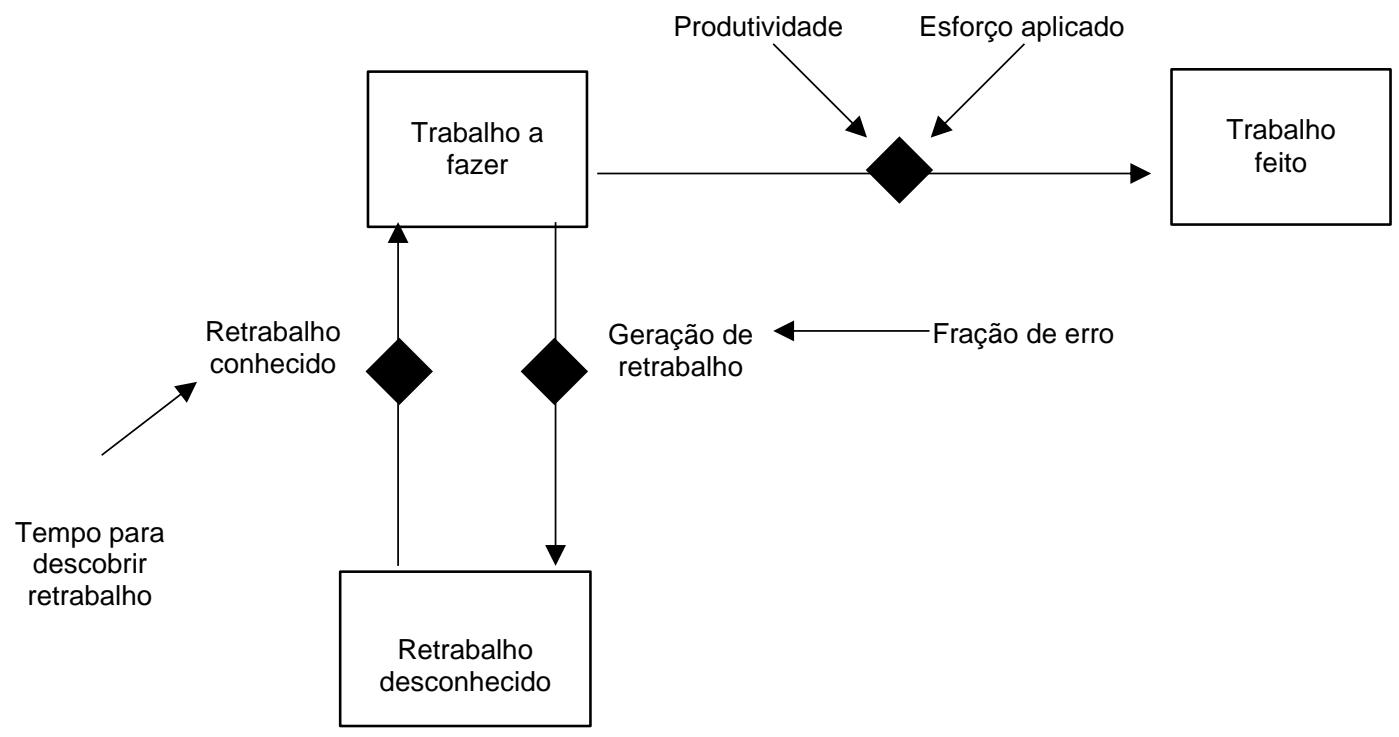

Outro estudo fundamental para justificar o uso de algumas variáveis endógenas e exógenas neste trabalho foi a revisão de Lyneis et al (2007), nela os autores contemplam diversos estudos que abordam sobre pontos chave que ampliam o ciclo de retrabalho e que mostram como outros autores inseriram novas perspectivas no mesmo, como por exemplo variáveis de controle de feedbacks (horas extras, paralelismo, etc.), que afetam a dinâmica do sistema na forma de laços negativos a fim de balancear o sistema.

Outra variável importante para o estudo também abordada na dinâmica de sistemas é a questão de riscos. Lyneis et al (2001) incorpora a variável risco de forma interativa e integrativa desde o pré-projeto ao pós-projeto, envolvendo gestão de riscos e gestão de mudanças, que segundo os autores, é uma parte da gestão de riscos, onde uma mudança, seja de escopo, ou design, invariavelmente poderá afetar o cronograma ou os custos do projeto, e consequentemente necessitará de alguma ação para balancear o sistema novamente.

Lyneis et al (2001) simula os impactos dos riscos durante a execução do projeto, e desta forma as mudanças necessárias a serem tomadas levando-se em consideração possíveis alterações no cronograma, sobreposição de fases, adição de pessoas, entre outras.

Além dos estudos relatados, outros trabalhos que auxiliam na utilização de algumas das variáveis deste trabalho, principalmente se falando de esforço são (Ford; Sterman, 2003; Yaghootkar; Gil, 2012; PMI, 2013). Neles, questões que abordam a força de trabalho são amplamente abordadas, como por exemplo, o 
paralelismo, o trabalho por meio de horas extras e a adição de mais pessoas.

Outros trabalhos corroboram com este estudo no que tange a projetos de inovação, sejam eles no desenvolvimento de novos produtos ou P\&D (Ottosson; Bjork, 2004; Lin; Chai; Wong; Brombacher, 2008; Akkermans; van Oorschot, 2016, etc.). Embora estes estudos abordem a dinâmica de sistemas em projetos de P\&D e desenvolvimento de novos produtos, os mesmos não tratam de variáveis exógenas que busquem inserir a variação no grau de inovação de projeto para projeto, além de uma entrada contínua de projetos e solicitações de cliente, o que pode influenciar substancialmente, por exemplo no aumento do escopo do projeto.

Com base nos estudos relatados, a base para este trabalho está estruturada e amplamente discutida na literatura, garantindo com isso a solidez que se necessita para demonstrar nos resultados a implicação da dinâmica destas variáveis, estoques e fluxos, somadas a novas variáveis, estoques e fluxos que ampliam o estudo da arte no que envolve projetos de inovação, principalmente em P\&D e desenvolvimento de novos produtos.

\section{PROCEDIMENTO METODOLÓGICO}

O presente estudo busca conhecimentos acerca do uso da dinâmica de sistemas aliada aos temas atraso em projetos, pesquisa e desenvolvimento (P\&D) no desenvolvimento de produtos inovadores. Para isso a pesquisa foi desenvolvida em 2 etapas. A primeira representa a pesquisa teórica, com 0 objetivo de identificar o estado da arte no que tange estes temas e embasar este estudo. A segunda etapa corresponde ao alinhamento da teoria e da prática, seguindo principalmente um método de pesquisa já difundido e conceituado na literatura a fim de garantir a correta utilização da dinâmica de sistemas neste trabalho. As duas etapas são explicitadas na sequência.

ETAPA 1. O estudo bibliométrico foi orientado pela busca de evidências dos temas principais deste trabalho, sendo eles: dinâmica de sistemas, gerenciamento de projetos, atraso em projetos e P\&D no desenvolvimento de novos produtos.

A primeira pesquisa foi realizada na Scopus no dia 04/06/2016 utilizando o seguinte filtro de palavras-chave: "dynamic systems" ou "systems dynamics". A amostra de resultados trouxe 60.550 estudos na base de dados Scopus e 17048 na base Web of Science (WOS). 
Após esse resultado, foi inserida a seguinte palavra chave para 0 prosseguimento da busca: "project management". Com isso a mesma trouxe os seguintes resultados: 732 trabalhos na Scopus e 15 na WOS.

Diante disso, foi inserida a palavra chave para o alinhamento com o tema atraso: "overlapping" ou "rework cycle". Com isso a mesma trouxe os seguintes resultados: 69 trabalhos na Scopus e 1 na WOS.

Por fim, foram inseridas as últimas palavra chave: " $R \& D$ " ou "research and development" ou "R \& D", ou "npd" ou "new product development”, resultando em 16 documentos para análise na Scopus e continuou com o mesmo número anterior da WOS.

A partir daí foram feitos os seguintes passos:

1. Inclusão dos "artigos" em uma ferramenta específica de análise (Mendeley), para exclusão dos possíveis duplicados, excluindo nesse caso o artigo da WOS, pois o mesmo já havia sido encontrado na base Scopus.

2. Análise do título e resumo para estabelecer conexão com os temas desejados, restando nesta etapa apenas 6 artigos com a respectiva aderência ao objetivo desse artigo.

3. Por fim, ao passar pelas duas primeiras análises, a última etapa foi a leitura completa do artigo para confirmar a aderência aos temas pesquisados, e a partir deste último ponto foi realizado então o último descarte, a fim de identificar quais dos estudos atendiam ao objetivo deste trabalho, onde foi constatado que todos os 6 artigos estavam aderentes aos temas desejados.

ETAPA 2. Esta etapa tem por finalidade alinhar a parte teórica, com a parte empírica, a fim de deixar o estudo mais aderente. Desta forma, a etapa foi seguir a metodologia desenvolvida por Sterman (2000), com intuito de deixar o trabalho o mais alinhado possível as boas práticas da dinâmica de sistemas. Sterman (2000) define uma modelagem para a dinâmica de sistemas em 5 passos, conforme mostra a figura 2. 
Fig. 2. O processo de modelagem (adaptado de Sterman, 2000)

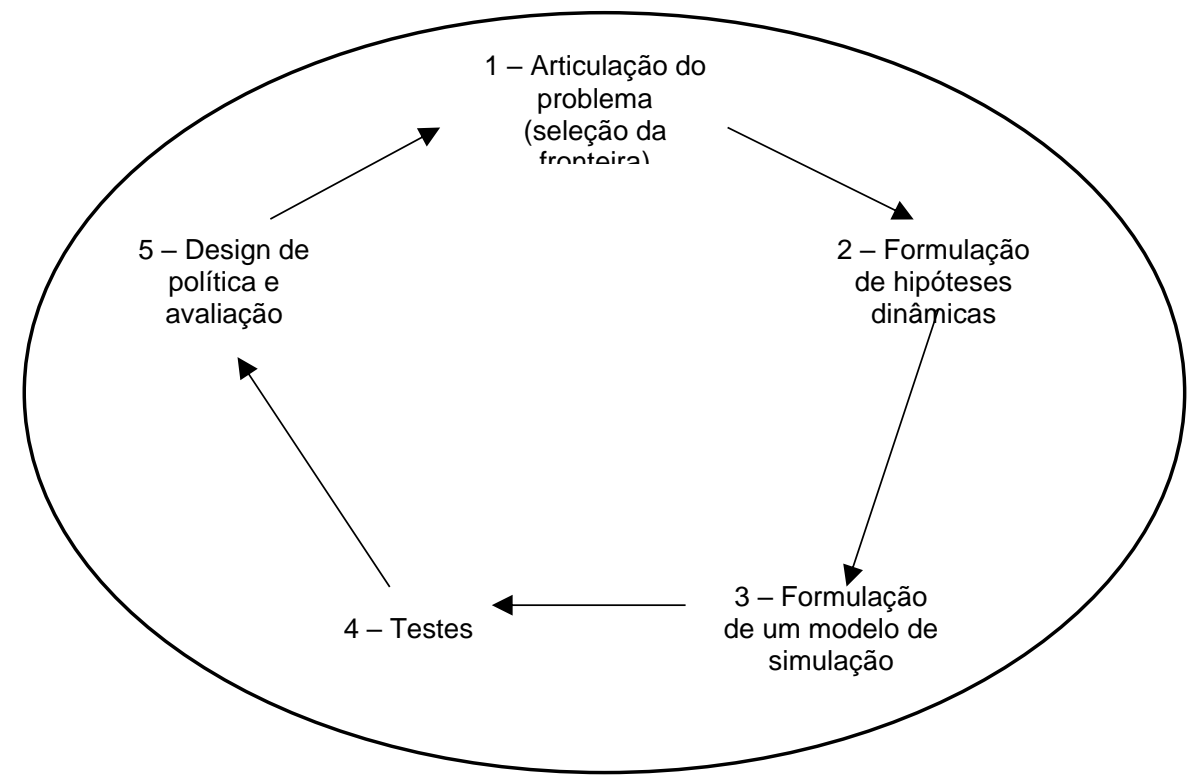

O primeiro passo do processo de modelagem é a definição do problema. Para este trabalho o problema definido foi o atraso nas entregas e projetos do instituto de inovação em questão, e quais seriam as variáveis do sistema que poderiam influenciar mais ou menos no mesmo. Neste passo foi verificado também a unidade de tempo e qual seria o universo de estudo para este trabalho, optando-se pelo levantamento de dados passados de 30 meses, já que este universo contemplava a quantidade necessária e mais realista para a modelagem.

No passo dois, são levantadas as hipóteses dinâmicas do sistema, e neste passo, partiu-se das seguintes hipóteses: 1) O sistema é influenciado significativamente devido ao grau de inovação dos projetos, 2) O sistema é significativamente influenciado pelo fator de aprovação de propostas e pela taxa de aprovação das solicitações dos clientes, 3) O atraso nas entregas e projetos se da principalmente devido a sua produtividade padrão e 4) A taxa de eficiência e a capacidade extra influenciam sobremaneira no sistema. Com base nessas hipóteses foram definidas as variáveis endógenas, exógenas, estoques e fluxos da modelagem, conforme mostra a tabela 1.

Tab. 1. Variáveis do sistema (elaborada pelos autores, 2016)

\begin{tabular}{|l|l|c|c|c|}
\hline \multicolumn{1}{|c|}{ Variável } & Endógena & Exógena & Fluxos & Estoque \\
\hline Solicitação de cliente & & $\mathrm{X}$ & & \\
\hline Taxa de aprovação de solicitação de cliente (SC) & & $\mathrm{X}$ & & \\
\hline Grau de inovação & & $\mathrm{X}$ & & \\
\hline Transformação de propostas & & $\mathrm{X}$ & & \\
\hline Propostas submetidas & & $\mathrm{X}$ & & \\
\hline
\end{tabular}




\begin{tabular}{|l|c|c|c|c|}
\hline Fator de aprovação de propostas & & X & & \\
\hline Fração de erro & & X & & \\
\hline Taxa de eficiência & & X & & \\
\hline Horas extras por pessoa & & X & & \\
\hline Produtividade & & $\mathrm{X}$ & & \\
\hline Propostas aprovadas & $\mathrm{X}$ & & & \\
\hline Solicitações aprovadas & $\mathrm{X}$ & & & \\
\hline Esforço aplicado & $\mathrm{X}$ & & & \\
\hline Capacidade extra & $\mathrm{X}$ & & & \\
\hline Contratar & $\mathrm{X}$ & & & \\
\hline Fator de conversão & $\mathrm{X}$ & & & \\
\hline Déficit de trabalho & $\mathrm{X}$ & & & \\
\hline Conhecimento trabalho restante & & & $\mathrm{X}$ & \\
\hline Entrada de propostas & & & $\mathrm{X}$ & \\
\hline Progresso & & & $\mathrm{X}$ & \\
\hline Geração de retrabalho & & & $\mathrm{X}$ & \\
\hline Retrabalho descoberto & & & $\mathrm{X}$ & \\
\hline Pessoas contratadas & & & $\mathrm{X}$ & \\
\hline Esforço nominal & & & & $\mathrm{X}$ \\
\hline Trabalho a fazer & & & & $\mathrm{X}$ \\
\hline Trabalho realizado & & & & $\mathrm{X}$ \\
\hline Retrabalho desconhecido & & & & $\mathrm{X}$ \\
\hline Pessoas & & & & \\
\hline
\end{tabular}

Para o passo três do modelo de Sterman (2000) tem-se a formulação do modelo de simulação. Nele se especificam as regras de estrutura de decisão, parâmetros, relações comportamentais, condições iniciais, entre outras. Sendo assim, com base nas variáveis do sistema, estoques e fluxos, desenvolveu-se 0 modelo da figura 3.

Fig. 3. O modelo de simulação (elaborado pelos Autores, 2016)

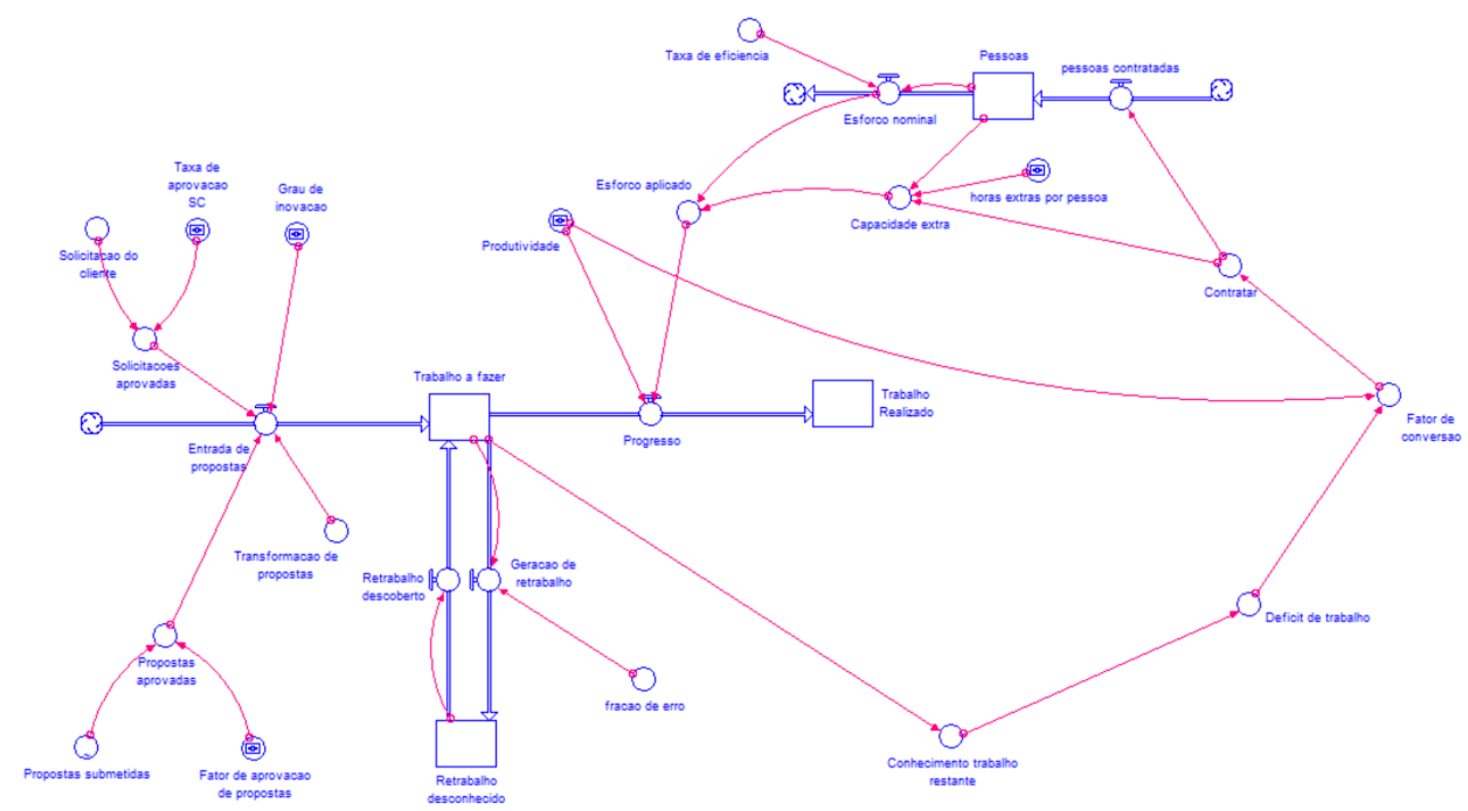


No passo quatro Sterman (2000) sugere que o modelo de simulação seja testado, de forma a comparar os modos de referência, verificar a robustez de condições extremas e a sensibilidade do modelo. Estes testes foram devidamente realizados, a fim de validar se o modelo estava robusto suficiente, sendo que os mesmos se mostraram adequados ao contexto aqui abordado.

Por fim, no passo cinco, o processo de modelagem insere a especificação de cenários, a concepção de políticas, análise de sensibilidade, questões como os efeitos das políticas assumidas, e as interações das mesmas, se há ou não sinergia entre elas. Este passo será verificado nos resultados e discussões com base em algumas hipóteses e simulações de alguns cenários feitos, a fim de validar estas hipóteses.

\section{RESULTADOS E DISCUSSÃO}

Para ampliar o estudo da arte que já é consolidado na união entre dinâmica de sistemas e gerenciamento de projetos, além de consolidar o modelo em questão a fim de confirmar ou não as hipóteses levantadas e o objetivo deste trabalho, se da a necessidade da execução e testes do modelo desenvolvido, como outros trabalhos nesta área assim também o fazem.

Desta forma, por meio de simulações de diversos cenários foram identificados resultados valiosos para o modelo, bem como, consequentemente para a tomada de decisões da empresa sobre estes cenários levantados.

O primeiro passo foi calibrar o modelo com o cenário mais realístico possível com base nos dados levantados, nas observações feitas na empresa, nas hipóteses identificadas e outras necessidades assumidas, e desta forma, a figura 4 mostra como o modelo se comportou em termos de trabalho a fazer, trabalho realizado, retrabalho desconhecido, capacidade extra e pessoas. Esta simulação base foi calibrada com suas variáveis principais da seguinte forma: Fator de aprovação de propostas = 30\%; Taxa de aprovação de solicitação de clientes (S.C) = 25\%; Pessoas = 24; Grau de inovação 50\%; Fator de erro = diretamente proporcional ao grau de inovação; Produtividade $=4$ pacotes de trabalho/mês por pessoa; Horas extras por pessoa/mês $=4$. 
Fig. 4. Estado inicial do sistema (elaborado pelos Autores, 2016)

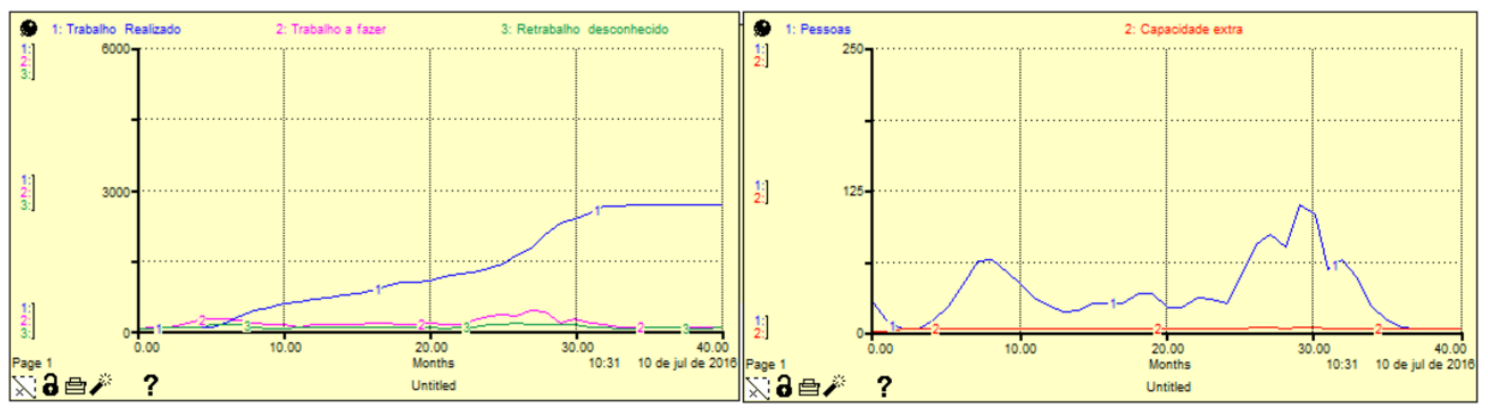

Para a simulação da figura 5 a única variável alterada foi o grau de inovação, que aumentou para 100\%, para verificar se o mesmo influenciava conforme a hipótese levantada.

Fig. 5. Aumento do grau de inovação (elaborado pelos Autores, 2016)

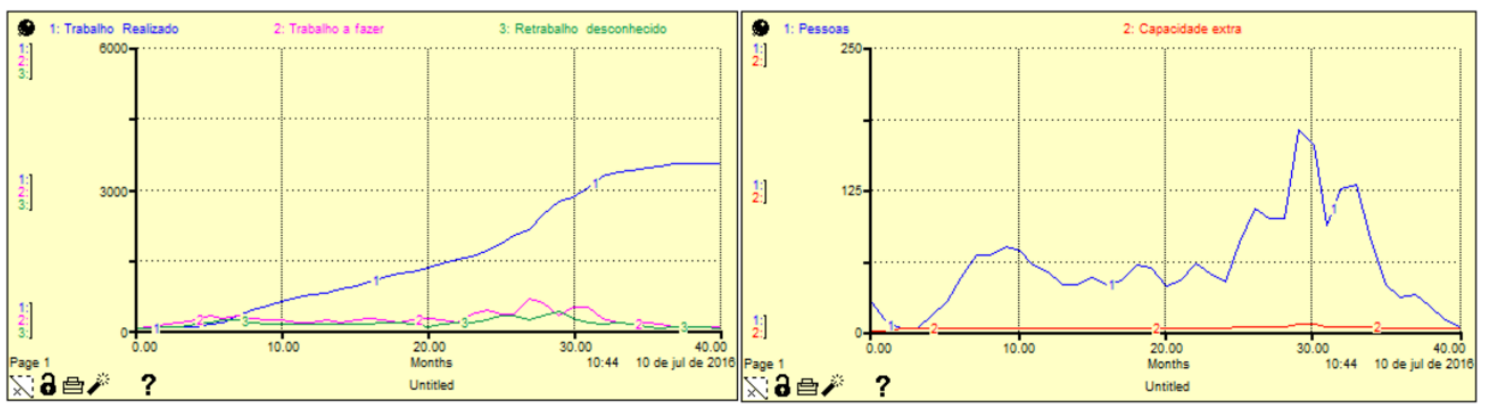

Na simulação da figura 6 as variáveis alteradas foram o fator de aprovação de propostas, que aumentou para 50\%, e a taxa de aprovação de solicitação de clientes, que aumentou para $50 \%$, a fim de verificar o quão as mesmas influenciavam no sistema.

Fig. 6. Aumento do fator de aprovação de propostas e taxa de aprovação de S.C (elaborado pelos Autores, 2016)

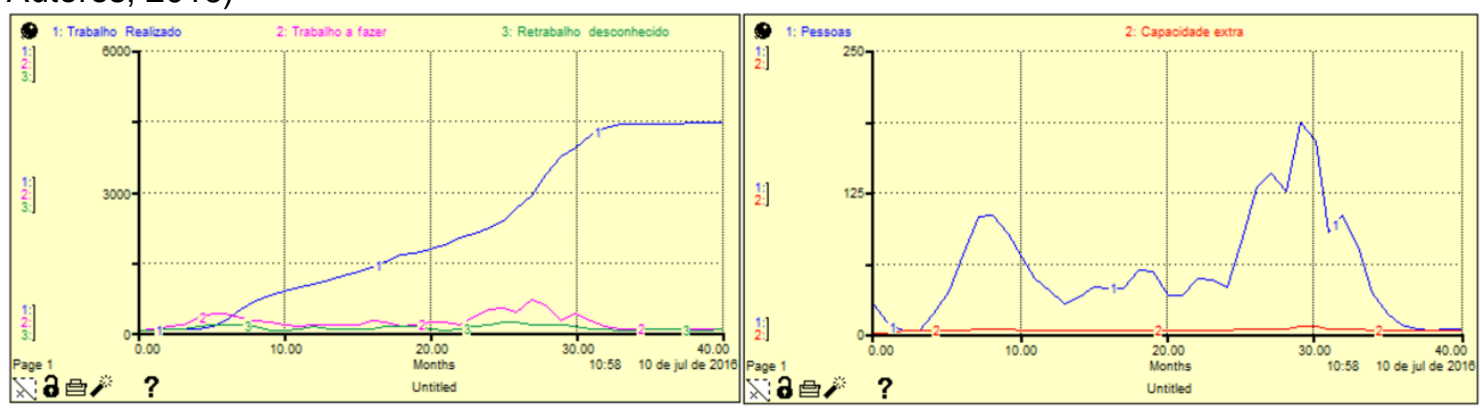

Para a simulação da figura 7 a variável alterada foi a produtividade, onde a mesma dobrou o seu valor, passando para 8 pacotes de trabalho/mês por pessoa. 
Fig. 7. Aumento da produtividade (elaborado pelos Autores, 2016)

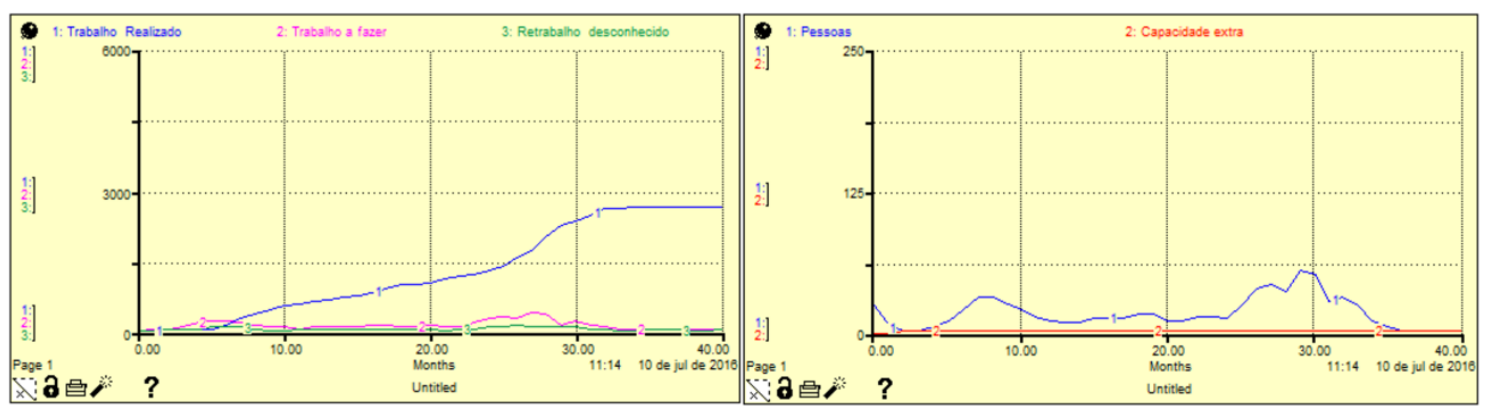

Por fim, para a simulação da figura 8 as variáveis alteradas foram a taxa de eficiência, que aumentou para 100\%, e as horas extras por pessoa/mês, que aumentou para 40 horas.

Fig. 8. Aumento da taxa de eficiência e horas extras (elaborado pelos Autores, 2016)

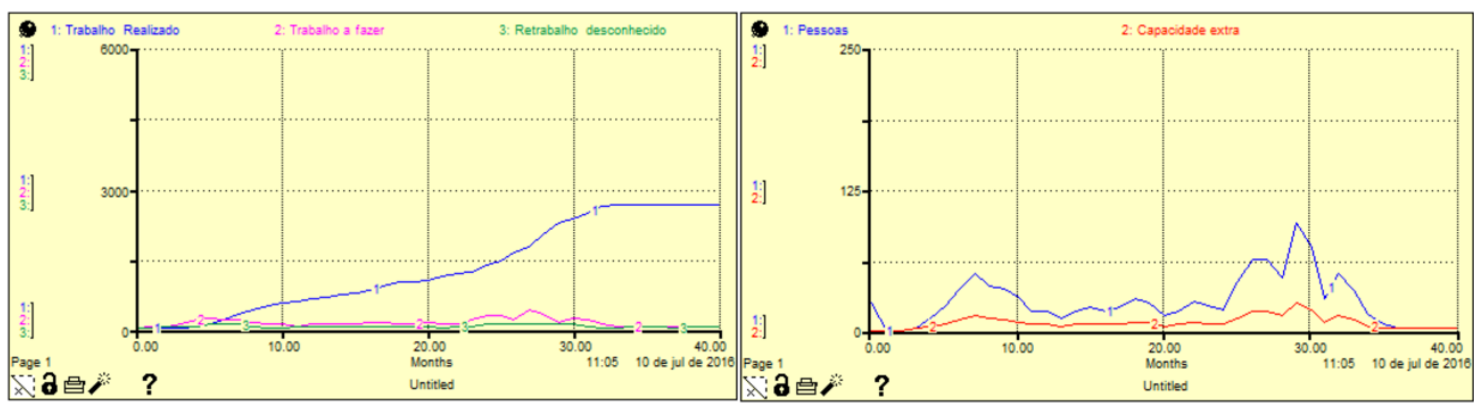

Conforme mostram as simulações feitas a fim de validar ou não as hipóteses levantadas, pode-se verificar que as principais variáveis que afetam o sistema negativamente são o fator de aprovação das propostas e o grau de inovação, onde as mesmas acabam gerando mais trabalho a fazer e retrabalho e maior necessidade de contratação de pessoas. Da mesma forma as variáveis que afetam o sistema positivamente são a produtividade e a taxa de eficiência, onde as mesmas acabam por reduzir o atraso no ciclo, além de necessitar bem menos contratações e horas extras a fim de resolverem o trabalho esperado.

Finalmente, tem-se também duas variáveis chave que não influenciaram significativamente a mudança base do sistema que foram a taxa de aprovação das solicitações do cliente, bem como a quantidade de horas extra/mês por pessoa. Estas variáveis se mostraram menos significativas que as demais, e no contexto estudado, foram as que menos geraram mudanças significativas na dinâmica do sistema. 


\section{CONCLUSÃO}

Conforme visto, os resultados encontrados foram significativos para explicitar os cenários das simulações do modelo a fim de possibilitar a tomada de decisões futuras mais assertiva e coesa. Pode-se verificar que as variáveis que mais influenciam no sistema modelado, e que acabam gerando cada vez mais retrabalho e mais atraso nas entregas e nos projetos são o fator de aprovação das propostas, o grau de inovação e a taxa de aprovação de solicitações de clientes.

Pode-se constatar também que conforme as quatro hipóteses levantadas para o modelo, a hipótese 1 de fato se confirmou conforme o esperado, onde aumentando-se o grau de inovação, maior será o fator de erro, o retrabalho, o atraso e a contratação. A segunda hipótese também se mostrou válida, em partes, especialmente quando o fator de aprovação de propostas aumenta, verificandose que o atraso e a contratação aumentam, mesmo o retrabalho não aumentando significativamente. A terceira hipótese da mesma forma verificou-se verdadeira no que tange a influencia positiva tanto na baixa do atraso, quanto na baixa significativa da contratação. Por fim a hipótese 4 mostrou que o que mais influencia o sistema é a taxa de eficiência, e não a quantidade de horas extras/mês por pessoa.

Isso mostra que quanto maior a demanda por projetos, e quanto mais inovadores são os mesmos, maior será o atraso, e mais o sistema tenderá a contratar pessoas e utilizar das horas extras para suprir a demanda, além de quanto maior a produtividade, menor o atraso e menos pessoas necessárias, neste contexto estudado. Além disso, este trabalho contribuiu para o estado da arte, principalmente nesses últimos dois pontos levantados, que até então não se mostraram devidamente tratados na literatura, demonstrando assim sua importância quando se analisa o ciclo de retrabalho em projetos e suas variações.

Como dificuldades encontradas, as principais foram a obtenção de alguns dados reais no que tange a pacotes de trabalho, produtividade e horas extras, que pudessem comprovar a robustez ainda maior do modelo, bem como, a pouca literatura que mostre detalhadamente modelos sobre o ciclo de retrabalho utilizados em projetos de P\&D e desenvolvimento de novos produtos.

Por fim, faz-se necessário como trabalhos futuros, estudos mais abrangentes e detalhados que insiram variáveis como o paralelismo, a mudança 
no prazo do projeto, fatores humanos, como turnover, motivação, entre outras, que integradas as variáveis aqui estudadas, possam ampliar e demonstrar ainda mais, a importância do uso da dinâmica de sistemas no gerenciamento de projetos. 


\section{REFERÉNCIAS}

Akkermans, H., \& van Oorschot, K. E. (2016). Pilot Error? Managerial Decision Biases as Explanation for Disruptions in Aircraft Development. Project Management Journal, 47(2), 79-102.

Cooper, K. G. (1980). Naval ship production: a claim settled and a framework built. Interfaces 10(6): 20-36.

Cooper, K. G. (1993). The rework cycle: why projects are mismanaged. PM network, 7(2), 5-7.

Eden, C., Williams, T., \& Ackermann, F. (1998). Dismantling the learning curve: the role of disruptions on the planning of development projects. International Journal of Project Management, 16(3), 131-138.

Eden, C. E, Williams T. M, Ackermann F. A, \& Howick S. (2000). On the nature of disruption and delay (D\&D) in major projects. Journal of the Operational Research Society 51(3), 291-300.

Ford, D. N., \& Sterman, J. D. (1998). Dynamic modeling of product development processes. System Dynamics Review, 14(1), 31-68.

Ford, D. N., \& Sterman, J. D. (2003). Overcoming the $90 \%$ syndrome: Iteration management in concurrent development projects. Concurrent Engineering, 11(3), 177-186.

Lin, J., Chai, K. H., San Wong, Y., \& Brombacher, A. C. (2008). A dynamic model for managing overlapped iterative product development. European Journal of Operational Research, 185(1), 378-392.

Lyneis, J. M., Cooper, K. G., \& Els, S. A. (2001). Strategic management of complex projects: a case study using system dynamics. System Dynamics Review, 17(3), 237-260.

Lyneis, J. M., \& Ford, D. N. (2007). System dynamics applied to project management: a survey, assessment, and directions for future research. System Dynamics Review, 23(2-3), 157-189.

Ottosson, S., \& Björk, E. (2004). Research on dynamic systems-some considerations. Technovation, 24(11), 863-869.

Project Manegement Institute (PMI). (2013). Guide to the project Management body of knowledge. Project Management Institute, Pennsylvania USA.

Reichelt, K., \& Lyneis, J. (1999). The dynamics of project performance: benchmarking the drivers of cost and schedule ove

rrun. European management journal, 17(2), 135-150.

Roberts, E. B. (1962). The dynamics of research and development (Doctoral dissertation, Massachusetts Institute of Technology). 
Sterman, J. D. J. D. (2000). Business dynamics: systems thinking and modeling for a complex world (No. HD30. 2 S7835 2000).

Williams, T., Eden, C., Ackermann, F., \& Tait, A. (1995). The effects of design changes and delays on project costs. Journal of the Operational Research Society, 46(7), 809-818.

Williams, T., Ackermann, F., \& Eden, C. (2003). Structuring a delay and disruption claim: An application of cause-mapping and system dynamics. European journal of operational research, 148(1), 192-204.

Yaghootkar, K., \& Gil, N. (2012). The effects of schedule-driven project management in multi-project environments. International Journal of Project Management, 30(1), 127-140.

Yi, T., \& Xiao, G. (2008, July). Applying System dynamics to analyze the impact of incentive factors' allocation on construction cost and risk. In 2008 International Conference on Machine Learning and Cybernetics (Vol. 2, pp. 676-680). IEEE. 